\title{
Sensory Stimulation for Nursing-Home Residents: Systematic Review and Meta-Analysis of Its Effects on Sleep Quality and Rest-Activity Rhythm in Dementia
}

\author{
Angela Joanna Prins ${ }^{a, b}$ Erik J. Anton Scherder ${ }^{b}$ Annemieke van Straten ${ }^{c}$ \\ Yvonne Zwaagstra $^{a}$ Maarten Valentijn Milders ${ }^{b}$ \\ ${ }^{a}$ Atlant (Elderly Care), Beekbergen, The Netherlands; ${ }^{b}$ Department of Clinical Neuropsychology, VU University \\ Amsterdam, Amsterdam, The Netherlands; ${ }^{C}$ Department of Clinical Psychology, VU University Amsterdam, \\ Amsterdam, The Netherlands
}

\section{Keywords \\ Sensory stimulation · Multi-sensory environments - Sleep \\ efficiency · Rest-activity rhythm · Circadian rhythm}

stimulation may prevent or improve sleep disturbances in nursing homes, and thereby contribute to a better quality of life for their patients.

(c) 2020 The Author(s) Published by S. Karger AG, Basel

\begin{abstract}
Introduction: Disrupted sleep-wake cycles might be associated with an exacerbation of behavioural disturbances and accelerate disease progression in dementia. The effect of sensory stimulation for improving sleep quality is unclear. Methods: A systematic literature search was performed and all studies examining the effects of a sensory stimulation intervention (i.e. bright light, massage, acupuncture, animalassisted interventions) on rest-activity rhythm (RAR) and/or nocturnal restlessness in nursing-home residents with dementia were included. Results: Sensory stimulation was shown to improve nocturnal behavioural restlessness as well as sleep duration and continuation, but the effect on the number of awakenings, RAR, and daytime sleep was negligible. Notable was the high heterogeneity between studies regarding treatments and patients' characteristics and sleep parameters. Conclusion: Sleep quality and nocturnal restlessness in nursing-home residents with dementia may benefit from sensory stimulation. An environment with sensory
\end{abstract}

karger@karger.com www.karger.com/dem

Karger ${ }^{\prime \prime} \div$

BOPEN ACCESS
(C) 2020 The Author(s)

Published by S. Karger AG, Basel

This article is licensed under the Creative Commons AttributionNonCommercial-NoDerivatives 4.0 International License (CC BYNC-ND) (http://www.karger.com/Services/OpenAccessLicense) Usage and distribution for commercial purposes as well as any distribution of modified material requires written permission.

\section{Introduction}

An estimated 50 million people worldwide live with dementia, and this number is expected to increase to 152 million by 2050 [1]. People with dementia prefer to live at home as long as possible, but institutionalization is often called for after the development of sleep disturbances and related behavioural and psychological symptoms (BPSD) such as anxiety, hallucinations, and aggression [2]. On average, sleep disturbances occur in 23\% (range $0-85 \%$ ) of people with dementia living in nursing homes, and residents with dementia using psychotropic drugs are more likely to experience sleep disturbances [3]. The severity of sleep disturbance increases with dementia severity, negatively affecting quality of life [4].

The most common sleep disturbances in nursinghome residents with dementia are difficulties falling or staying asleep, waking up too early in the morning, and 
excessive daytime sleepiness [5]. These disturbances can lead to disrupted sleep-wake cycles (the rest-activity rhythm) $[5,6]$. Sleep disturbances and a disrupted restactivity rhythm (RAR) are among the most distressing behaviours for people with dementia, their relatives, and caregivers $[7,8]$. Sleep quality is a requirement for wellbeing in people with dementia $[4,9]$ and fragmented sleep is closely related to cognitive decline (e.g., memory impairment), neuropsychiatric disorders (e.g., depression), functional decline, and morbidity and mortality in dementia [10-12]. Therefore, it is important to treat sleep disturbances in nursing-home residents who have dementia, in order to improve their sleep quality and RAR, which may reduce other BPSD and improve their quality of life $[4,13]$.

Treating sleep disturbances with sleep medication (usually benzodiazepine derivatives) can have adverse effects, such as confusion, and also exacerbates daytime sleepiness and night-time awakenings [14]. The preferred method to improve sleep in dementia, cognitive behavioural therapy [15], appears effective in mild dementia [16] but is not compatible with the cognitive and physical capacities of people with moderate-to-severe dementia [11]. Instead, stimulating residents during the day with activities that fit their individual cognitive and physical capacities can be helpful in regulating RAR $[17,18]$.

Sensory stimulation has become increasingly popular in nursing homes and appears to be a promising approach for ameliorating mood disturbances and behavioural problems in moderate-to-severe dementia [19-21]. Sensory stimulation involves stimulating one (uni-sensory stimulation [USS], e.g. light therapy) or more (multi-sensory stimulation [MSS], e.g., Snoezelen ${ }^{\circledR}$ ) primary senses [22], and aims to prevent sensory deprivation by creating an enriched environment in a non-directive manner [18]. Sensory stimulation is based on the assumption that the primary sensory systems (i.e., visual, auditory, olfactory, gustatory, and tactile) can still be activated in the advanced stages of dementia [23,24]. Sensory stimulation is the only person-centered approach which can be implemented by adjusting the nursing-home environment and is suitable for people with severe dementia [25]. Sensory stimulation may also work as a "Zeitgeber" to improve sleep disturbances and RAR in dementia [6].

However, to date, evidence for its effectiveness is scarce and contradictory [26-28]. Possible explanations for the lack of evidence are the high heterogeneity between studies regarding the target population, intervention characteristics (e.g., duration and intensity), and methodology. The aim of this systematic review and meta-analysis, with

\begin{tabular}{|c|c|c|}
\hline P & Participants & Dementia \\
\hline I & Intervention & Sensory stimulation \\
\hline C & Comparisons & Standard care/no intervention \\
\hline O & Outcome & $\begin{array}{c}\text { Sleep quality, circadian rhythm, sleep } \\
\text { disturbances }\end{array}$ \\
\hline
\end{tabular}

Fig. 1. PICO search model.

extensive subgroup analysis, was to examine the effectiveness of sensory stimulation on the sleep quality and RAR of nursing-home residents with dementia, with a focus on different uni-sensory components.

\section{Methods}

\section{Search Strategy}

A systematic literature search was conducted on December 3, 2019. The PICO search model was used to identify research terms (Fig. 1). Studies were extracted from; PubMed, The Cochrane Library, PsycINFO, Embase, and CINAHL. The search terms differed slightly between the different databases. For an overview, see the online supplementary Material (for all online suppl. material, see www.karger.com/doi/10.1159/000509433). The review is registered with the International Prospective Register of Systematic Reviews (PROSPERO; registration No. 104104).

\section{Study Selection}

After completing the literature search, duplicates were removed. All remaining studies were selected by 2 independent reviewers according to the inclusion and exclusion criteria (Table 1). We screened title and abstract first and full text second. Disagreements between the authors were discussed after the first selection and again after the second selection until agreement was reached.

\section{Quality Assessment}

The criteria from the Cochrane Collaboration tool (CCT) [29] and the Risk of Bias in Nonrandomized Studies of Interventions (ROBINS-I) tool [30] were used to assess the methodological quality of randomized controlled trials (RCTs) and non-RCTs, respectively. Risk of bias was ranked as low, moderate, high, or no information in RCTs, and low, moderate, serious, critical, or no information in non-RCTs. Eighteen studies also reported outcomes other than sleep, e.g., mood [31-48]. For these studies, the quality assessment of some domains of the CCT and ROBINS-I (e.g., blinding of participants and outcome assessment, missing data, and selective reporting) was based only on sleep and rest-activity outcomes, i.e., actigraphy data and observational data. All selected studies were evaluated independently by 2 authors. Differences in assessment between these authors were resolved by consensus.

\section{Outcomes}

The primary and secondary outcomes were measured using wrist-worn activity monitors (Actiwatch). Primary outcomes were RAR and nocturnal awakening. RAR was described by 3 
Table 1. Inclusion and exclusion criteria for systematic search

\begin{tabular}{ll}
\hline Inclusion criteria & Exclusion criteria \\
\hline $\begin{array}{l}\text { Articles including experimental research design; RCT, non-RCT, } \\
\text { quasi experimental, cross-over design, pre-post studies with control }\end{array}$ & $\begin{array}{l}\text { Other publication types than articles including reviews or published } \\
\text { comments/replies on articles, single case studies, without control }\end{array}$ \\
\hline Publication year 1990-2019 & Publication year <1990 \\
\hline Articles written in English & Articles not written in English \\
\hline Human studies & Animal studies \\
\hline $\begin{array}{l}\text { Diagnosis of dementia (all subtypes) according to DSM-IV/V and } \\
\text { NINCDS-ADRDA or ICD-10 }\end{array}$ & $\begin{array}{l}\text { Other neurologic diseases (Korsakov, MS, Parkinson, Huntington) } \\
\text { or no clear diagnosis }\end{array}$ \\
\hline $\begin{array}{l}\text { Intervention study; sensory based and applied to individuals or in a } \\
\text { group performed by staff members of researchers (not family caregivers) } \\
\text { at a nursing home }\end{array}$ & $\begin{array}{l}\text { No intervention study or combinations of pharmacological interventions } \\
\text { with sensory stimulations. Intervention performed by family caregivers } \\
\text { at home or daycare }\end{array}$ \\
\hline Sensory stimulation interventions/multisensory environments & $\begin{array}{l}\text { Other forms of stimulation (psychopharmacological and medical } \\
\text { treatments including DBS and EEG }\end{array}$ \\
\hline $\begin{array}{l}\text { Referring to sleep and/or circadian rhythm disturbances } \\
\text { Standardized outcome measures }\end{array}$ & \begin{tabular}{l} 
Outcomes other than sleep \\
\hline
\end{tabular} \\
\hline
\end{tabular}

nonparametric variables [49]; relative amplitude (RA), interdaily stability (IS), and interdaily variability (IV). RA was calculated as the (normalized) difference between the means of the most active 10 -h period (M10) and the least active 5 -h period (L5) in the average 24 -h pattern [49]. Higher values indicated a larger difference between daytime activity and nocturnal rest, and thus a stronger rhythm (range $0-1$ ). IS was a measure of the degree of resemblance between activity patterns over multiple days [49]. Higher values indicated a more stable rhythm (range $0-1)$. IV represents fragmentation of periods of rest and activity [49]. Higher values indicated a more fragmented rhythm (range $0-2$ ). Nocturnal awakening was described by 2 variables; the number of nocturnal awakenings (NA) and wake after sleep onset (WASO, i.e., minutes awake during the night after sleep onset).

The secondary outcomes were total sleep time (TST) night (duration of sleep at night in hours [between 8 p.m. and 8 a.m.]), TST day (duration of daytime sleep in hours from wake up to bedtime), sleep efficiency (SE; the proportion of actual sleep time in hours compared with hours lying in bed during the night) and sleep onset latency (SoL; minutes awake after going to bed).

The tertiary outcomes were sleep disturbance observations (SDO) and sleep quality observations (SQO) during the night (8 p.m. to 8 a.m.). SDO was measured with the Neuropsychiatric Inventory - night-time behaviour (NPI-K) [50], the Nursing Home Behaviour Problem Scale [51], the Behavioural Pathology in Alzheimer's Disease Rating Scale - activity disturbance (Behave-AD) [52], and sleep diaries recording behavioural restlessness. SQO was measured with the Pittsburgh Sleep Quality Index (PSQI) [53], Behave-AD - diurnal rhythm scale [52], and the Depressive Symptom Assessment for Older Adults - sleep impairment and cyclic function scales (DSAOA) [54]. In addition, sleep diaries were used about daytime sleepiness, early morning awakenings, awakenings during the night, and nocturnal sleep time, and were based on observations by nursing staff.

Sensory Stimulation and Sleep in Dementia
Main Effects

Data analysis was performed using Comprehensive MetaAnalysis $^{\complement}$ v3.0 [55]. Effect sizes for all sleep outcomes with a control group were calculated with a between-group Hedges' $g$ and 95\% confidence intervals (CIs). For studies with a pre-post or cross-over design, a within-group Hedges' $g$ and 95\% CIs were calculated. We examined the effects as observed immediately after a maximum 3-month intervention (post-test). Follow-up effects $>1$ week after intervention completion were not examined as few studies reported them.

To calculate pooled effect sizes and standard errors, we extracted the reported means, standard deviations (SDs), and number of participants for each group (intervention and control group) for each sleep measurement. Each study provided only 1 effect size. For studies with $\geq 2$ intervention groups and 1 control group (as was the case in 4 studies [45, 56-59]), the intervention groups were combined into a single intervention score by pooling means, SDs, and sample sizes using the formulae proposed in the Cochrane Handbook [60]. When means and SD were missing, effect sizes were calculated using $p$ values, sample sizes, and correlations. When exact $p$ values were missing, we assumed a $p$ value of 0.5 for non-significant (ns) results and 0.05 for significant results. We estimated effect sizes under the conservative assumption of a prepost correlation of 0.7 [61]. Mean effect sizes were calculated according to the random-effects model. Heterogeneity was tested with the $I^{2}$ statistic, the Q test, and the examination of funnel plots.

Publication bias was tested with Egger's test [62] and by inspecting the funnel plot. If the Egger's test was significant (e.g., $p<0.05$, one-sided), the estimated effect sizes were adjusted for publication bias by using the trim-and-fill procedure of Duval and Tweedie [63] with the random-effects model.

\section{Subgroup Effects and Regression Analysis}

Subgroup analyses were performed for the outcomes related to RAR and nocturnal behavioural disturbances (IV, IS, RA, WASO, 


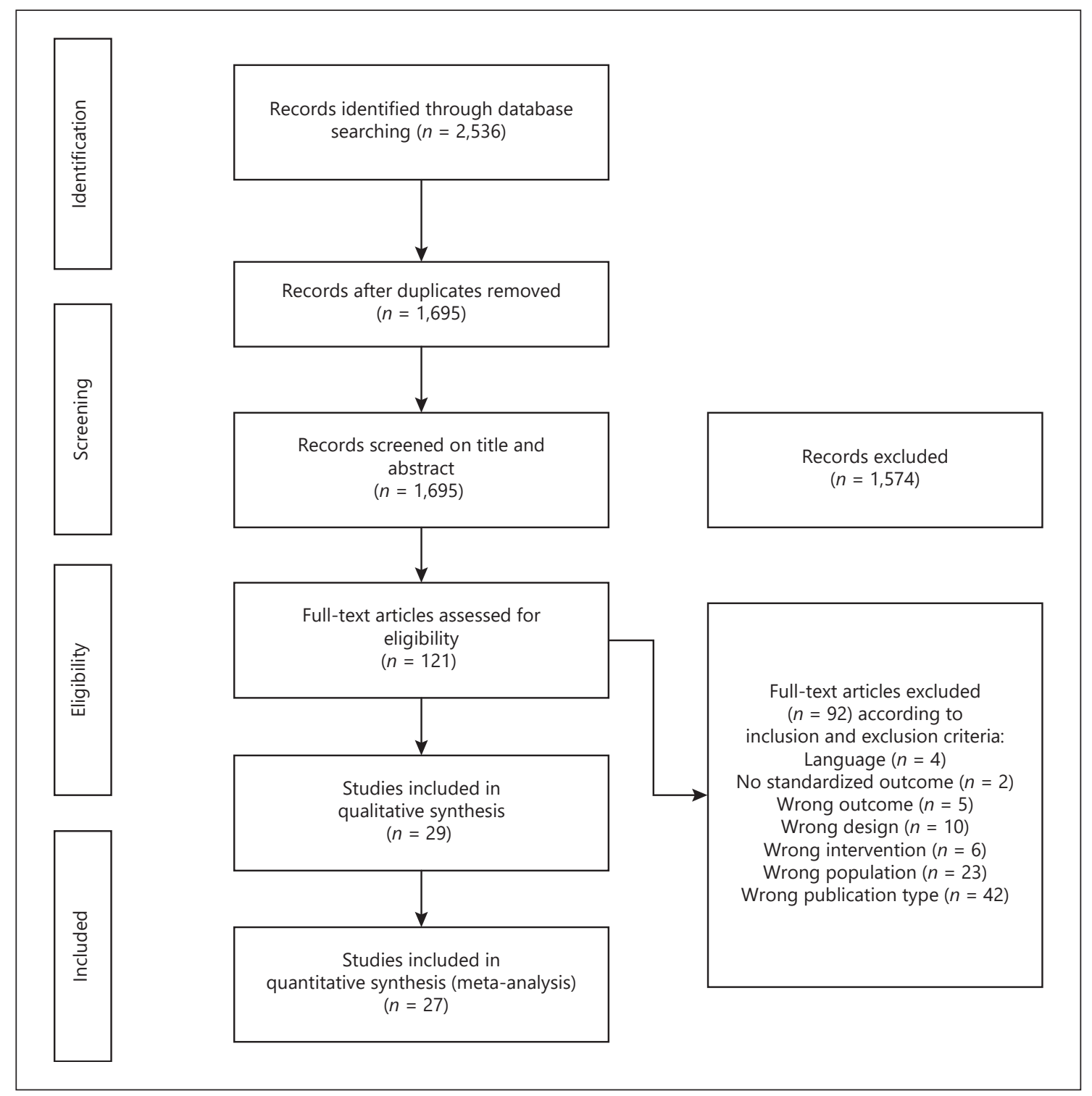

Fig. 2. Flow diagram of literature search.

SoL, and SDO). For these outcomes, we examined the effect of differences in study design and quality, intervention type, and disease comorbidity (sleep disturbances, agitation, and depression) on reported effect sizes. A mixed-effects model was used in the subgroup analyses. Pooled effect sizes within subgroups were calculated with the random-effects model, and the fixed-effects model was used to test the difference between subgroups. Several metaregression analyses were performed to examine the relationship between study characteristics (e.g., disease duration in hours and weeks and lux used in light interventions) and population characteristics (sex, dementia severity, and dementia type) and the combined effect size across all studies. The $p$ values were corrected for the number of subgroup ( $p \leq 0.0125)$ and regression $(p \leq 0.008)$ analyses.

\section{Results}

\section{Study Selection}

After the first search, 2,536 references were extracted from PubMed (462), The Cochrane Library (102), PsycINFO (224), Embase (1,499), and CINAHL (249). After removing duplicates, 1,695 references were screened based on title and abstract. Disagreement (a rate of $3 \%$ ) between the reviewers was discussed and resulted in the selection of 121 articles. After full-text screening, disagreement (a rate of 6\%) between the reviewers was discussed, and 29 studies were included for further analysis, 


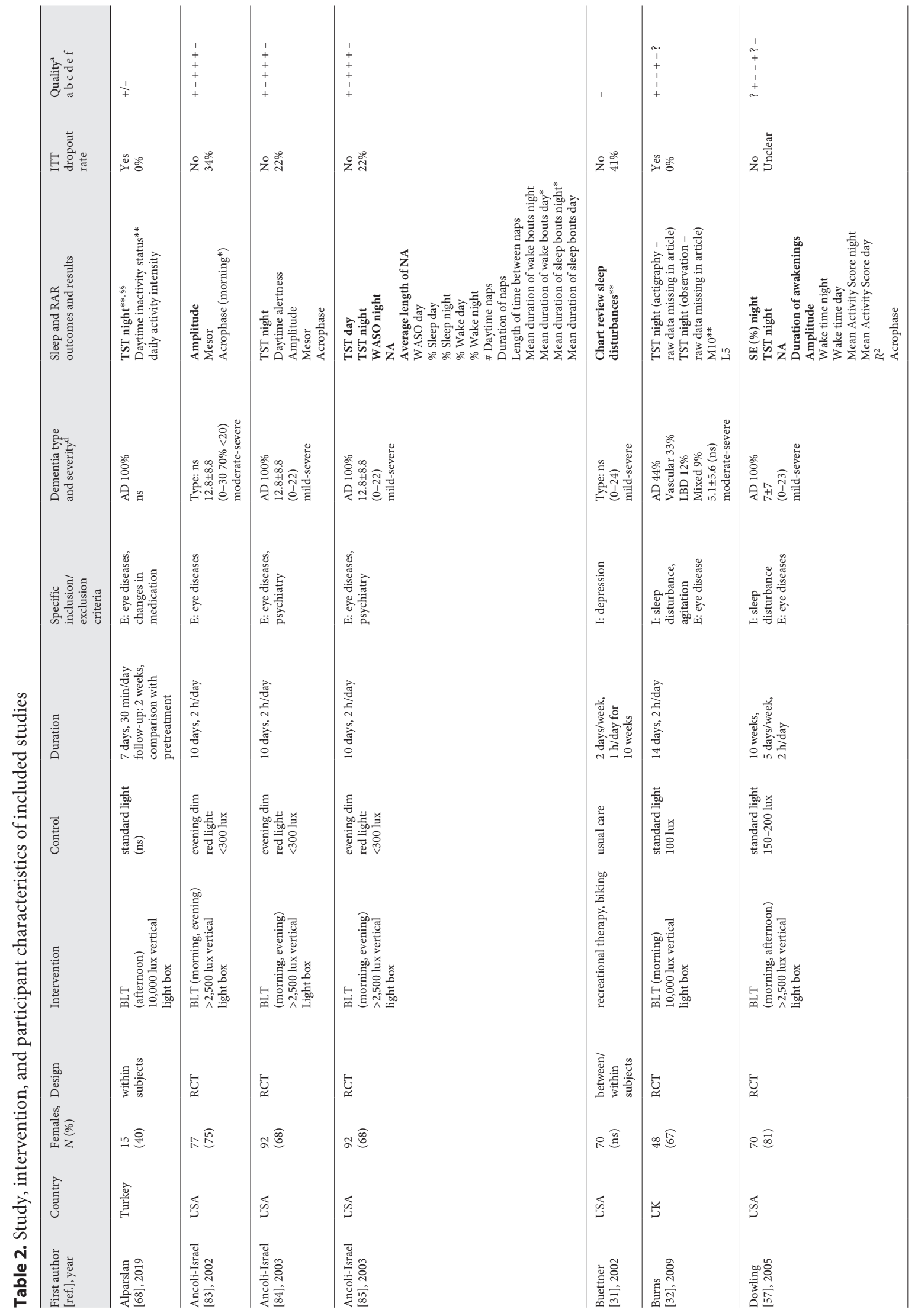




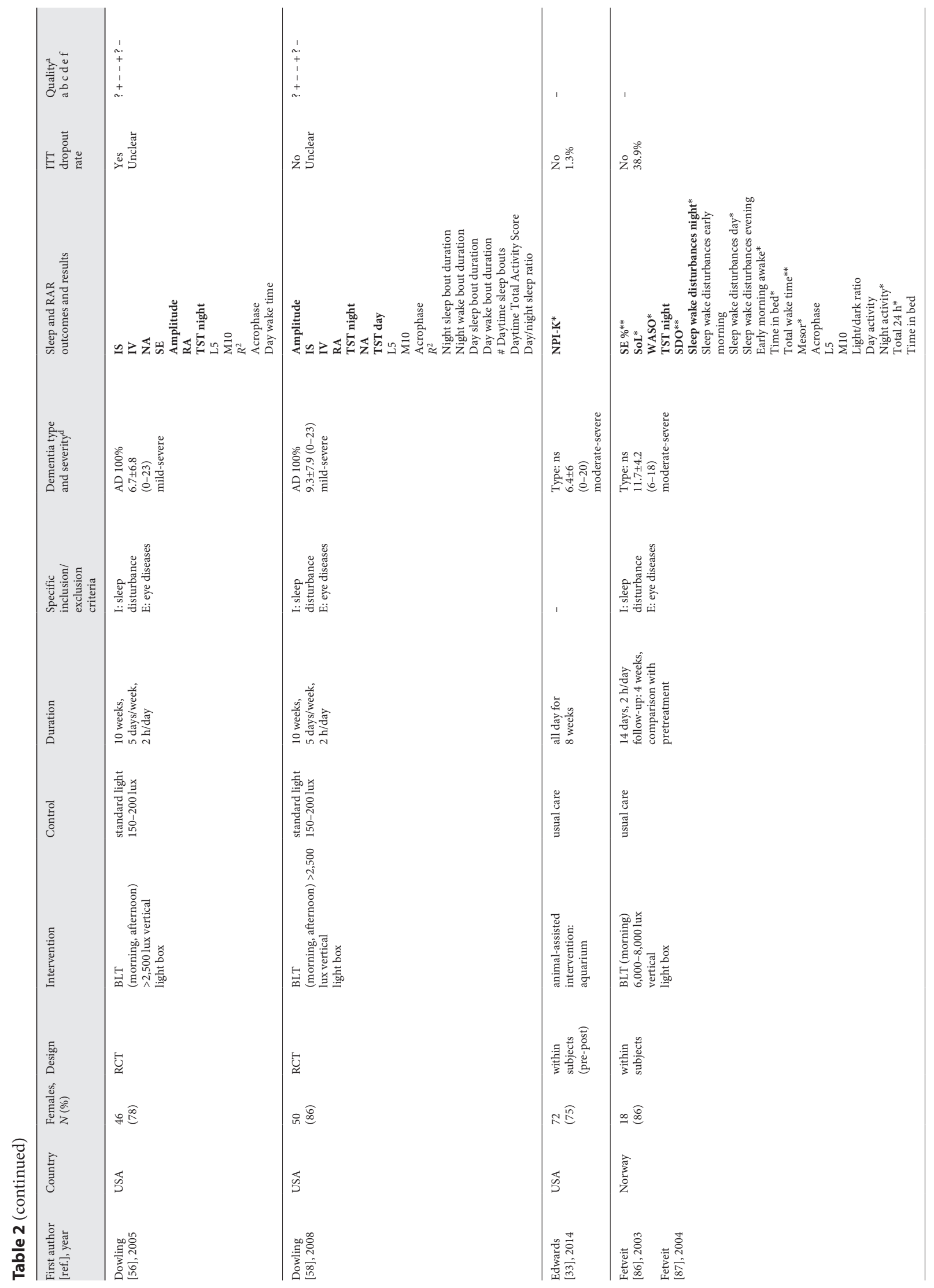




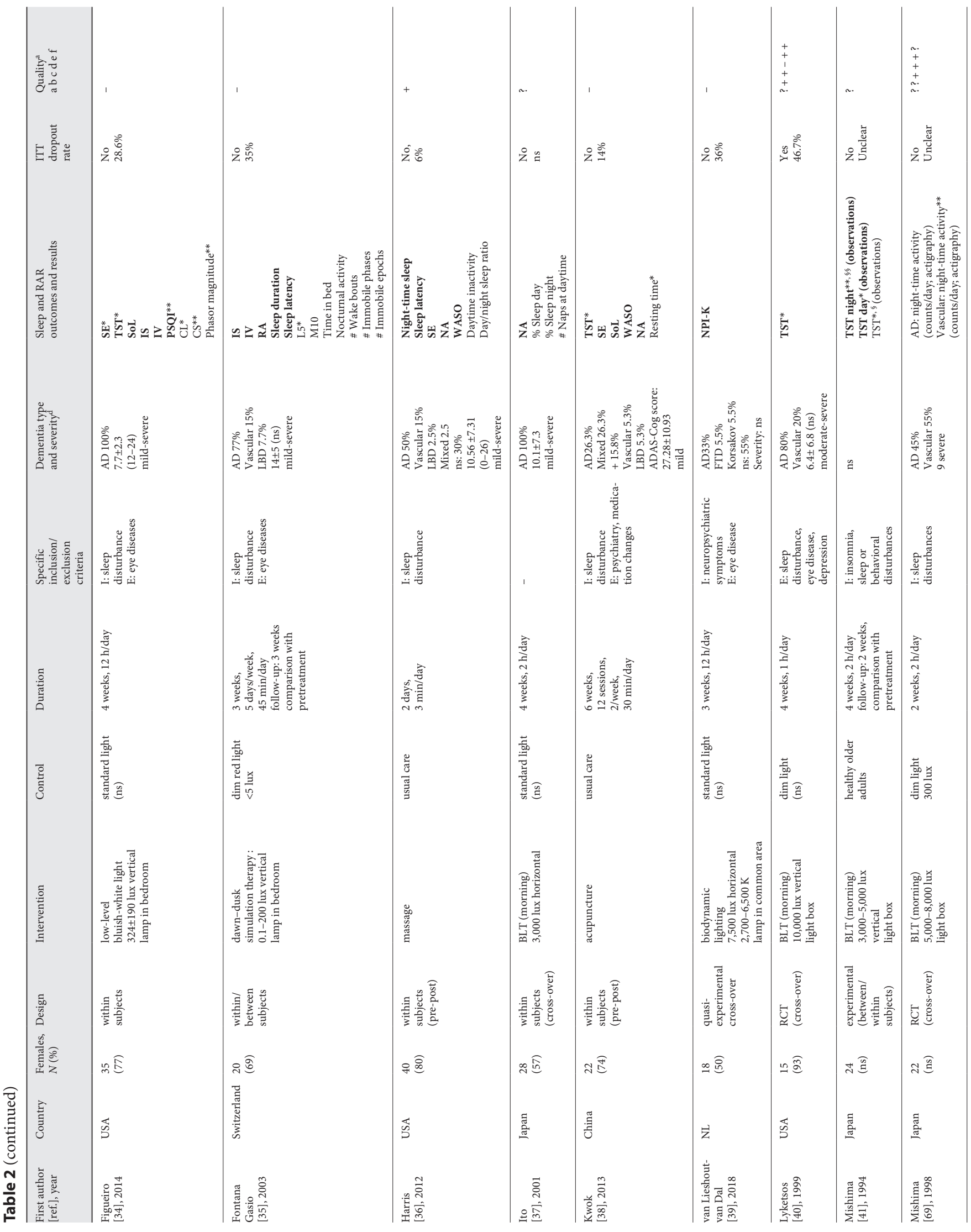




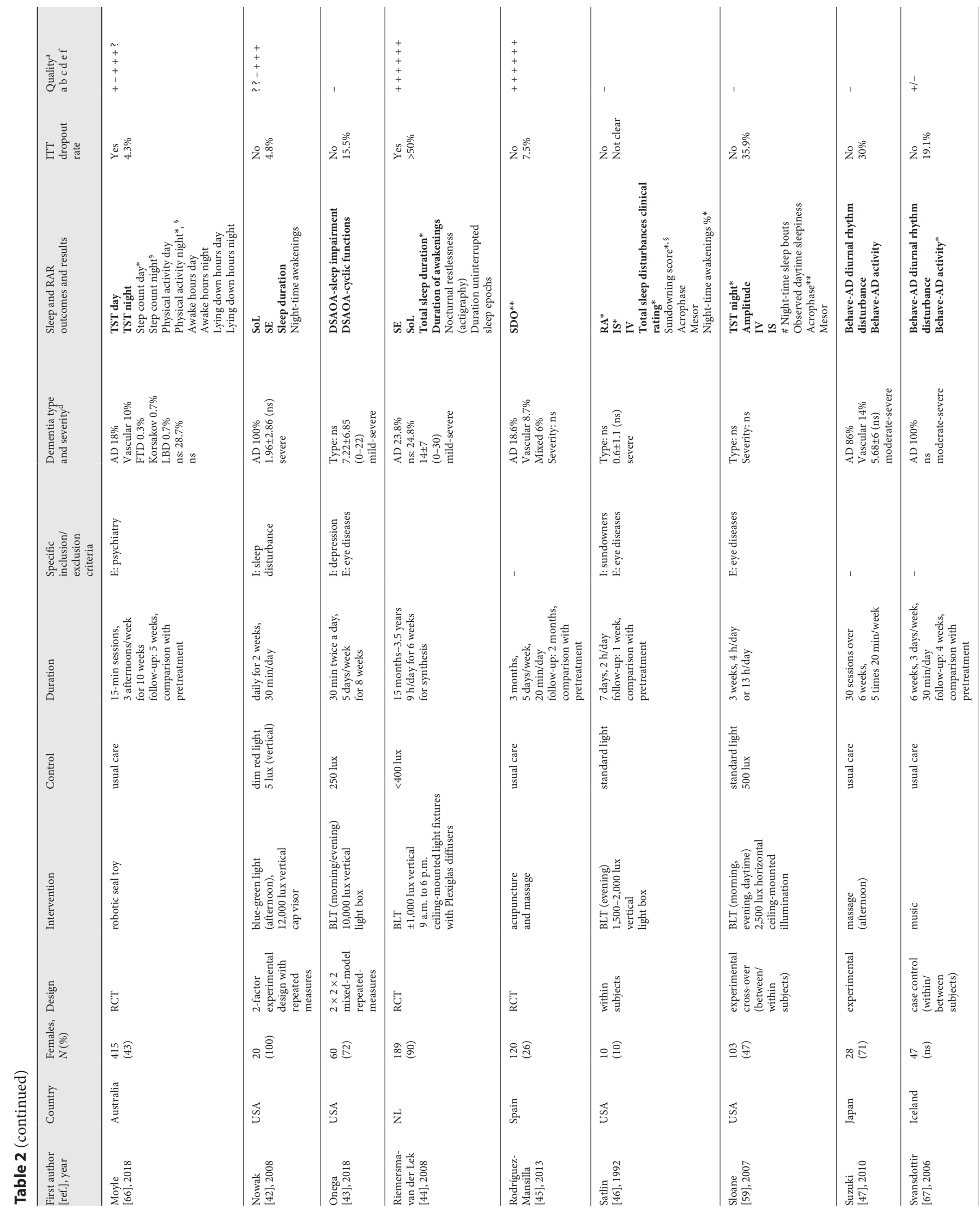




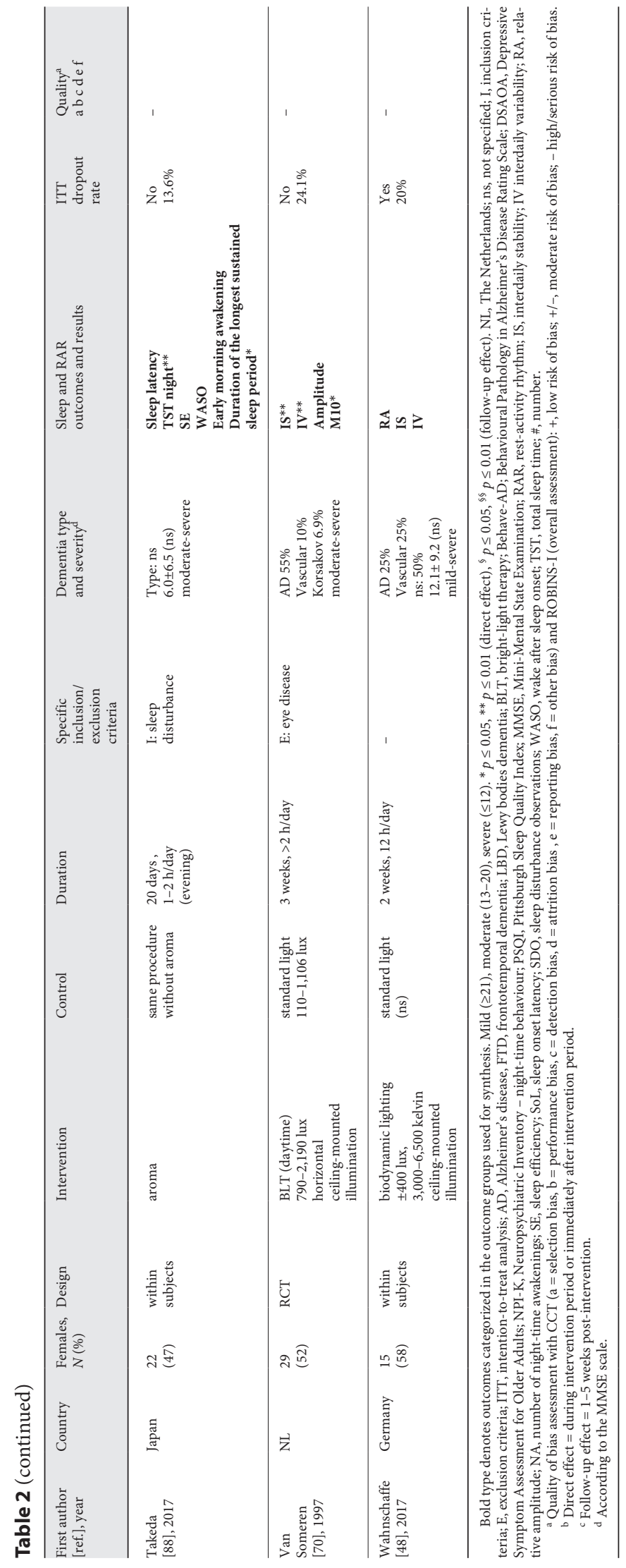

including 27 for synthesis. Figure 2 presents a flow diagram of the search results according to the PRISMA guidelines [64].

\section{Study Characteristics}

Table 2 represents details of the study characteristics. The 29 studies included a total of 1,717 nursing-home residents with dementia, ranging between 10 and 415 participants per study. Ten studies used a RCT design. Eight studies used a non-RCT design with a control group and 11 studies used a pre-post design. Most studies were from the USA, followed by Europe and Asia.

Ten to $100 \%$ of the participants within each study were females. Dementia types were as follows: $18-100 \%$ of the participants were diagnosed with $\mathrm{AD}, 10-33 \%$ with vascular dementia, $0.7-12 \%$ with Lewy bodies dementia, $0.3-5.5 \%$ with frontotemporal dementia, and 2.5-9\% with mixed dementia. Dementia severity was measured in 946 participants (55\% of the total sample), with a mean Mini Mental State Examination (MMSE) [65] score between 0.6 and 12.8, which corresponds to moderate-tosevere dementia. Comorbid sleep or mood disorders at the time of inclusion were diagnosed in 401 (23\% of the total sample) and 148 ( $9 \%$ of the total sample) participants, respectively. TST night (i.e., 8 a.m. -8 p.m.), measured with actigraphy in 851 participants (50\% of the total sample), was $7.4 \mathrm{~h}$ (95\% CI 4.8-11.9 h). In the remaining 866 participants (50\%), the severity of sleep disturbances preintervention was not reported. Participant dropout rates during the intervention period ranged from 0 to $50 \%$.

Two studies applied MSS as outside recreational activities and robotic toy therapy [31, 66] 2-3 times/week for 10 weeks. Other studies applied USS as light $(n=20)$, massage $(n=3)$, acupuncture $(n=2)$, music $(n=1)$, animal-assisted $(n=1)$ and aromatherapy $(n=1)$. Regarding light therapy, 15 studies applied bright light $(1,000-$ 12,000 lux vertical), 3 studies applied a high light intensity but lower than bright light ( $<750$ lux vertical), and 2 studies applied biodynamic lighting (400-750 lux horizontal, 2,700-6,500 K). Most studies $(n=13)$ placed light boxes in front of the resident with dementia for $0.5-2 \mathrm{~h} /$ day. Seven studies examined the effect of ceiling-mounted illumination or independent lamps in a common room for all-day exposure. The dosage and duration of the light interventions varied between $30 \mathrm{~min}$ and $13 \mathrm{~h}$ per day for 1 week to 15 months. In most studies, light exposure started between 7.00 or 10.00 a.m. [46]. Fourteen of the 20 studies $(70 \%)$ excluded patients with severe visual disturbances. 


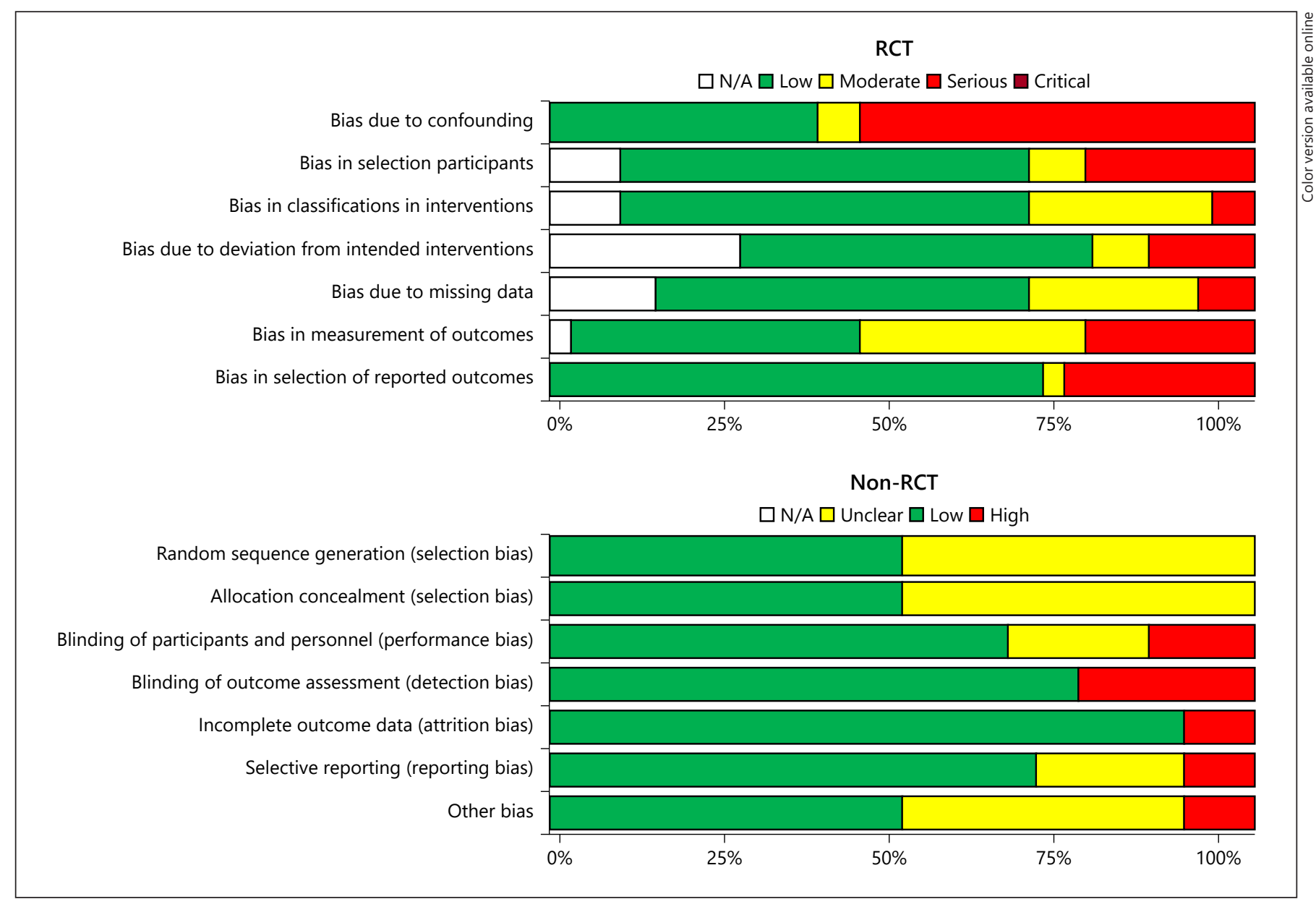

Fig. 3. Graphical representation of risk of bias in RCTs and non-RCTs included in the qualitative analysis.

\section{Risk of Bias within Studies}

Disagreement between the reviewers of risk of bias based on the CCT and ROBINS-I tool were discussed (RCTs: disagreement rate $22.6 \%$; non-RCTs: $43.8 \%$ ) and resolved (Fig. 3). Only 3 studies were judged to have a low risk of bias $[36,44,66]$ and 2 a moderate risk of bias [67, 68]. The remaining studies were evaluated as having a high/serious/critical risk of bias $(n=16)$, or else varied between a low and high risk of bias $(n=5)$ depending on outcome. In 3 studies [37, 41, 69], there was not enough information to evaluate the risk of bias.

\section{Synthesis of Results: Main Effects}

Twenty-seven studies $(n=1,238,72 \%)$ were available for synthesis (Fig. 2). One study [32] was excluded because of missing raw data. USS ( $n=1,006,59 \%)$ had significant small positive effects on RAR combined, RA, TST night, and SE. USS had significant small negative effects on WASO and SoL (Table 3) and a significant medium negative effect on SDO and SQO. IS/IV, NA, SoL, and TST day did not significantly postintervention compared to usual care. The heterogeneity within most of the outcomes varied between medium and large. MSS $(n=226,13 \%)$ had no significant effect on any of the outcome variables $[31,66]$.

\section{Additional Analysis: Differences between Study,} Intervention, and Patient Characteristics

Regarding the observations by nursing staff (subjective outcomes), the combined effect size was medium with medium heterogeneity. When considering actigraphic measurements (objective outcomes), the combined effect size was low with medium heterogeneity (Table 4). Effect sizes of the combined sleep outcomes did not differ between subjective and objective measurement methods $(Q=0.71, p=0.40, \mathrm{nc}=29)$. The combined effect size of RCTs was medium with medium heterogeneity. 
Table 3. Main post-test effects of USS

\begin{tabular}{|c|c|c|c|c|}
\hline Outcomes & $\mathrm{Nc}(\mathrm{Np})$ & Hedges' $g(95 \%$ CI $)$ & $I^{2}(95 \% \mathrm{CI})$ & NNT \\
\hline \multicolumn{5}{|l|}{ Primary outcomes } \\
\hline \multicolumn{5}{|l|}{ Rest-activity rhythm } \\
\hline RA & $7(268)$ & $0.37 *(0.07$ to 0.67$)$ & $59^{*}(5$ to 82$)$ & 4.85 \\
\hline IS & $6(174)$ & $0.39(-0.14$ to 0.92$)$ & $86^{* *}(71$ to 93$)$ & 4.59 \\
\hline IV & $6(174)$ & $-0.63(-1.29$ to 0.03$)$ & $90^{* *}(80$ to 95$)$ & 2.91 \\
\hline RAR (combined) & $8(278)$ & $0.41^{*}(0.02$ to 0.80$)$ & $78^{* *}(57$ to 89$)$ & 4.39 \\
\hline \multicolumn{5}{|l|}{ Nocturnal awakenings } \\
\hline WASO & $8(413)$ & $-0.29 *(-0.54$ to -0.04$)$ & $70^{* *}(37$ to 85$)$ & 6.17 \\
\hline \# Awakenings & $5(184)$ & $-0.17(-0.43$ to 0.08$)$ & $4(-388$ to 81$)$ & 10.42 \\
\hline \multicolumn{5}{|l|}{ Secondary outcomes } \\
\hline TST night & $15(593)$ & $0.38^{* *}(0.22$ to 0.54$)$ & $44(-4$ to 70$)$ & 4.72 \\
\hline SE & $9(350)$ & $0.48^{* *}(0.24$ to 0.72$)$ & $50 *(-7$ to 78$)$ & 3.76 \\
\hline SoL & $8(280)$ & $-0.25^{*}(-0.46$ to -0.05$)$ & $33(-51$ to 70$)$ & 7.14 \\
\hline TST day & $2(106)$ & $-0.04(-0.22$ to 0.13$)$ & $0(-2,854,734$ to 100$)$ & 45.45 \\
\hline \multicolumn{5}{|l|}{ Tertiary outcomes } \\
\hline SDO & $6(276)$ & $-0.57^{* *}(-0.96$ to -0.12$)$ & $67^{* *}(22$ to 86$)$ & 3.18 \\
\hline SQO & $7(221)$ & $-0.61^{* *}(-1.02$ to -0.20$)$ & $71^{* *}(38$ to 87$)$ & 2.99 \\
\hline Actography combined & $19(840)$ & $0.39^{* *}(0.21$ to 0.56$)$ & $63^{* *}(39$ to 77$)$ & 4.59 \\
\hline Observations combined & $11(473)$ & $-0.63^{* *}(-0.92$ to -0.35$)$ & $70^{* *}(44$ to 84$)$ & 2.91 \\
\hline All outcomes combined & $25(1006)$ & $0.47^{* *}(0.31$ to 0.63$)$ & $64^{* *}(45$ to 76$)$ & 3.85 \\
\hline RCTs & $8(476)$ & $0.67^{* *}(0.30$ to 1.05$)$ & $77^{* *}(39$ to 83$)$ & 2.75 \\
\hline
\end{tabular}

RAR, rest-activity rhythm; RA, (relative-) amplitude; IS, interdaily stability; IV, interdaily variability; WASO, wake up after sleep onset; TST, total sleep time; SE, sleep efficiency; SoL, sleep (onset) latency; SDO, sleep disturbance observations; SQO, sleep quality observations; RCTs, randomized controlled trials; CI, confidence interval; Nc, number of comparisons; Np, number of participants; NNT, number needed to treat. ${ }^{*} p \leq 0.05,{ }^{* *} p \leq 0.01$.

Subgroup analysis revealed significantly higher effect sizes for IV $(Q=14.61, p \leq 0.01)$, IS $(Q=19.34, p \leq 0.001)$, and SDO $(\mathrm{Q}=5.30, p \leq 0.05)$ in RCTs versus non-RCTs. In studies with a low/moderate risk of bias, the effect sizes were significantly higher for WASO $(Q=13.64, p \leq 0.001)$ and SDO $(Q=9.79, p \leq 0.01)$ when compared to studies with a high/serious risk of bias. No significant subgroup differences were found for other sleep outcomes. No significant differences in sleep parameters were found for intervention type and inclusion or exclusion criteria. For details of the effect sizes and heterogeneity within each group, see online supplementary Additional file 2.

Meta-regression analysis revealed no significant relationship between the effectiveness of USS on sleep and any of the following predictors: gender, dementia severity (MMSE score), intervention duration (h/week and total of weeks), intervention dosage (lux of light interventions). For details of the coefficients, see Table 5. The effect sizes did not differ between the different dementia types $(Q=3.82, p=0.58, \mathrm{nc}=22)$ and dropout rates $(Q=$ $4.22, p=0.52, \mathrm{nc}=22)$.

Sensory Stimulation and Sleep in Dementia

\section{Publication Bias}

Visual inspection of the funnel plots and execution of Egger's test indicated possible publication biases for IV $\left(t_{4}=2.79, p \leq 0.05\right)$ and TST night $\left(t_{13}=2, p \leq 0.03\right)$. According to the trim-and-fill procedure [63], 5 studies measuring IV $(n=1)$ and TST night $(n=4)$ were missing.

When the estimated missing effect sizes were imputed using the trim-and-fill algorithm [63], adjusted effect sizes for IV ( $g=0.77,95 \%$ CI $0.11-1.43$ ) became larger and significant, and for TST night ( $g=0.28,95 \%$ CI $0.12-0.43$ ) the adjusted effect size became smaller but remained significant.

\section{Discussion}

The aim of this review and meta-analysis was to examine the effectiveness of MSS and USS on sleep quality and RAR. USS including visual and tactile stimulation were effective for improving sleep quality and RAR (effect size 0.47). More specifically, visual and tactile stimulation for 


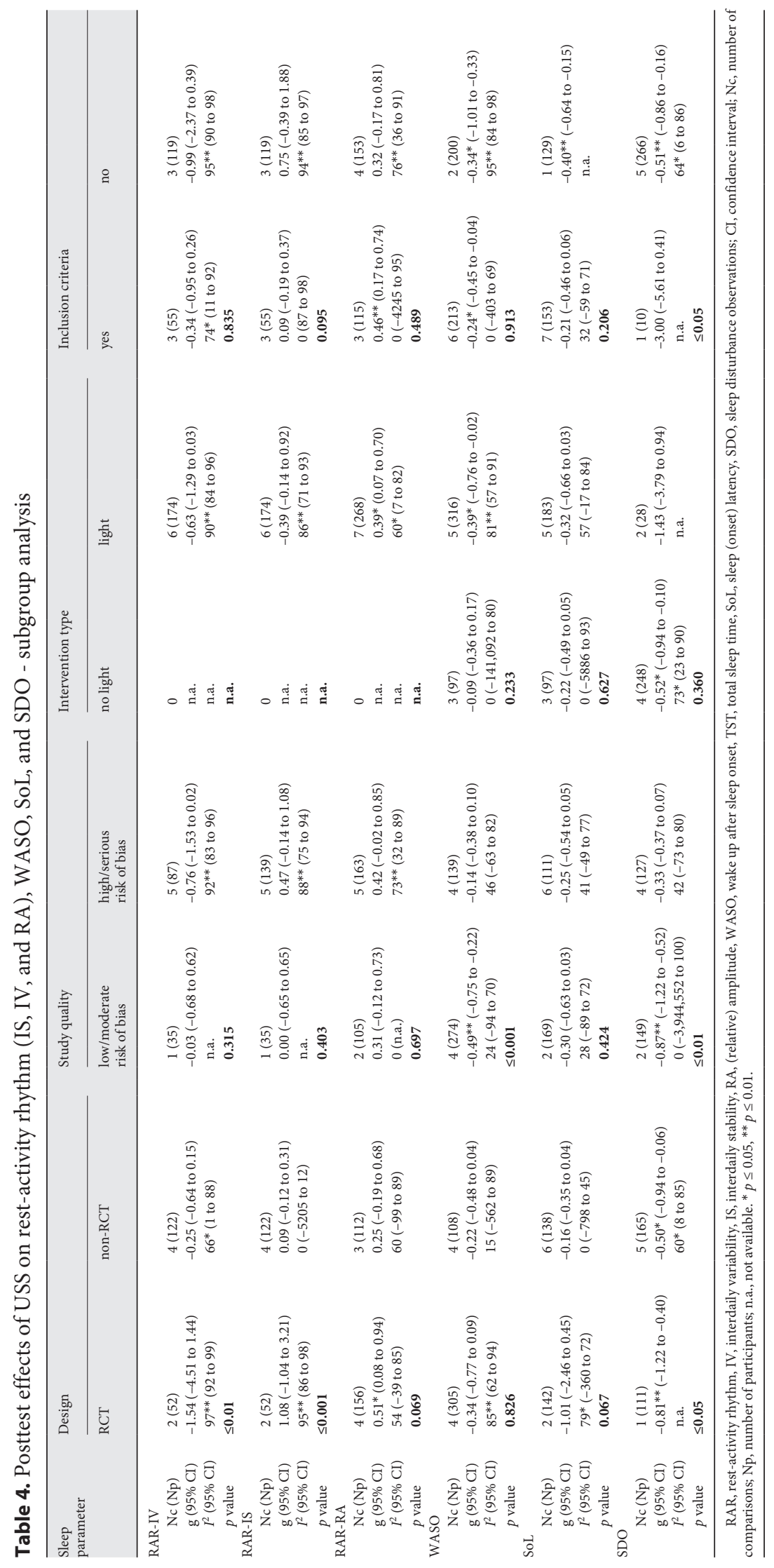


Table 5. Regression coefficients of characteristics of the interventions and target population

\begin{tabular}{lllll}
\hline Predictor & B & 95\% CI & SE & $p$ value \\
\hline Female gender, \% & 0.75 & -0.42 to 1.92 & 0.60 & 0.209 \\
Dementia severity (MMSE mean score) & 0.01 & -0.05 to 0.06 & 0.03 & 0.743 \\
Intervention duration (h/week) & 0.00 & -0.0 .00 to 0.00 & 0.00 & 0.581 \\
Intervention duration (total weeks) & -0.04 & -0.06 to -0.10 & 0.04 & 0.299 \\
Intervention dosage (lux) & 0.00 & -0.00 to 0.00 & 0.00 & 0.624 \\
\hline
\end{tabular}

There were 13 comparisons and 482 patients (28\% of the total sample).

a minimum of $30 \mathrm{~min} /$ day for 2 weeks was found to effectively reduce NA, SoL, and sleep disturbance, and improve nocturnal sleep duration and SE. Only 2 studies examined MSS in relation to sleep quality [31, 66], but none examined its effect on RAR. In the following paragraphs, we discuss the effects of USS on RAR and sleep quality for varying study characteristics.

Rest-Activity Rhythm. USS using light therapy (a minimal intensity of 790 lux) was only effective in strengthening the circadian rhythm (RA) in residents without severe eye diseases and in RCTs with a high risk of bias [46, 70], but did not improve fragmentation in the 24-h RAR (IV) or IS over multiple days. The effect on the RA could be attributed to dementia severity and study quality. The 2 studies with positive effects included only residents with moderate-to-severe dementia [46, 70]. More severe circadian rhythm disturbances are associated with more severe dementia, leaving more room for improvement. Reliable measurement of RA, IS, and IV requires $>7$ days of actigraphic recording [71], which was not achieved in 4 of the 7 studies $[38,46,58,70]$.

Sleep Quality. Consistent with recent reviews $[26,72,73]$, our meta-analysis revealed significant positive effects of USS using light therapy or acupuncture on TST night. Only light therapy was effective in improving SE and SoL, and decreasing the duration of awakenings, although the effects were small. Previous meta-analyses [74, 75] and reviews $[73,76]$ examining light therapy found mixed results regarding TST, SoL, early morning awakening, and daytime sleepiness. In all reviews and meta-analyses, the short-term effects on SE (\%) and continuation (WASO) were robust, especially in samples of nursing-home residents with moderate-to-severe dementia [76]. This supports evidence for the effectiveness of visual and tactile stimulation to improve sleep quality in nursing-home residents with dementia.

Night-Time Restlessness. Nursing staff observed less behavioural restlessness during the night when residents with dementia received light therapy, acupuncture, massage therapy, and animal-assisted interventions. The effects were medium to large, as in previous reviews $[26,56-58,74,76$, 77], and larger in RCTs with a low risk of bias than in those with a high risk of bias. No differences in effects were found between dementia patients with and dementia patients without a diagnosis of mood and/or sleep disorders. It remains a challenge to evaluate the efficacy of USS on nighttime restlessness of patients with or without mood and/or sleep disorders because in most articles, the severity of sleep disturbances preintervention was not described clearly.

Factors Affecting the Efficacy of Sensory Stimulation. In contrast to a previous meta-analysis [74], dementia severity, dementia type, gender, treatment duration, and light intensity (lux values) were not related to the effectiveness of sensory stimulation. One reason could be that, in the selected studies, only $28 \%$ of the data were available for analysis, due to the incomplete description of interventions and the characteristics of participants. Consequently, the statistical power of the meta regression analyses was limited. Dropout rates during the intervention periods were normally distributed between the studies and no association with effect sizes was found, suggesting that the effects of dropout on the results were limited affected.

In summary, sensory stimulation (in particular, visual and tactile stimulation) can have a small to medium positive effect on the duration of nocturnal awakenings, SE, and SoL, and thereby improve the total sleep duration and quality. We speculate that higher arousal during the day, in combination with a higher circadian amplitude, might lead to longer uninterrupted sleep during the night. These positive effects were observed in both actigraphic results and behaviour observations by nursing staff. This is an important and clinically relevant finding because nocturnal awakenings, in particular, can be a precursor for disorientation, anxiety, wandering during the night, and waking up other residents, all of which were reported as distressing for nursing staff $[4,7,8,78]$. Because improvements in sleep quality can be beneficial for both 
nursing-home residents and staff, implementing sensory stimulation interventions in nursing homes is recommended. In addition, treatment of nocturnal awakenings might be valuable in preventing further deterioration of behavioural disturbances and cognitive decline $[10,79]$.

This meta-analysis has several limitations. First, we did not control for the timing aspect of sensory interventions, while the daily routine and optimal timing may stabilize the circadian rhythm on its own [80]. Second, because of the small sample size and the large differences in sleep outcomes, measurement instruments, and procedures between studies, the results of the subgroup and regression analyses should be interpreted with caution. However, the extensive subgroup analysis is a valuable addition to earlier reviews and meta-analyses.

In order to make the studies more comparable and increase the level of knowledge about improving sleep in nursing-home residents with dementia, it is important to set up a standard protocol for researchers to apply the same intervention and measuring instruments. Based on this review and meta-analysis and past research, we recommend the use of both actigraphy (especially nonparametric variables like RA, IS, and IV [81]) and standardized observation scales to measure sleep disturbances in dementia (e.g., NPI-K or Behave-AD subscales: activity and diurnal rhythm disturbances [82]). More research using RCTs with sufficient methodological quality is needed to examine the effect of MSS and USS on the sleep quality and RAR in nursing-home residents with dementia with or without comorbid sleep disturbances, and to examine the differences between sensory stimulation interventions with or without light therapy. In this way, establishing implementation guidelines for nursing homes and analyzing the costs and benefits of different types of sensory stimulation interventions will become possible in the future.

\section{Conclusion}

The purpose of this study was to examine the effectiveness of MSS and USS on the RAR and sleep quality of nursing-home residents with dementia, while focusing on the different uni-sensory components. This is the first systematic review and meta-analysis which has investigated the high heterogeneity across studies with regard to interventions and patient characteristics. We found that sensory stimulations like bright light, massage, acupuncture, and animal-assistant interventions are effective for reducing nocturnal awakenings, SoL, and SDO, and improving nocturnal sleep duration and SE. Clinically relevant is the possibility that longer sleep duration and continuation might be associated with less agitated and aggressive behaviour during the night. To strengthen the RAR, only bright-light therapy seemed effective. The effect of MSS on the rest-activity rhythm and sleep quality remained unclear. Despite its exploratory nature, this study offers valuable insights into the understanding and treatment of sleep disturbances in nursing-home residents with dementia and points out directions for future research.

\section{Statement of Ethics}

The review is registered with the International Prospective Register of Systematic Reviews (PROSPERO; registration No. 104104).

\section{Conflict of Interest Statement}

The authors have no conflicts of interest to declare.

\section{Funding Sources}

This article was written in collaboration with Atlant (elderly care), Beekbergen and VU University Amsterdam, The Netherlands.

\section{Author Contributions}

A.J.P. made substantial contribution to the conception, design, acquisition, analysis and interpretation of data and drafted the entire manuscript. E.J.A.S., M.V.M and Y.Z. contributed to designing the study, interpreting the data and revising the manuscript for important intellectual content. A.S. contributed to the methods and data-analysis and was revolved in revising the manuscript for important intellectual content. All authors read and approved the final manuscript.
References
1 Prince M, Wimo A, Guerchet M, Ali G, Wu Y, Prina M. World Alzheimer Report 2015. The global impact of dementia. An analysis of prevalence, incidence, cost \& trends. London: Alzheimer's Disease International; 2015. World Health Organ-ization [Cited April 15, 2016]. Available from: https://www alz co uk/research/World AlzheimerReport2015 pdf. 2017.

2 Toot S, Swinson T, Devine M, Challis D, Orrell $\mathrm{M}$. Causes of nursing home placement for older people with dementia: a systematic review and meta-analysis. Int Psychogeriatr. 2017 Feb;29(2):195-208.

3 Wilfling D, Dichter MN, Trutschel D, Köpke S: Prevalence of Sleep Disturbances in German Nursing Home Residents with Dementia: A Multicenter Cross-Sectional Study. J Alzheimers Dis. 2019;69(1):227-36.
Prins/Scherder/van Straten/Zwaagstra/ Milders 
4 Petrovsky DV, McPhillips MV, Li J, Brody A, Caffeé L, Hodgson NA. Sleep disruption and quality of life in persons with dementia: A state-of-the-art review. Geriatr Nurs. 2018 Nov;39(6):640-5.

5 Bliwise DL. Sleep in normal aging and dementia. Sleep. 1993 Jan;16(1):40-81.

6 Van Someren EJ. Circadian and sleep disturbances in the elderly. Exp Gerontol. 2000 Dec; 35(9-10):1229-37.

7 Taemeeyapradit U, Udomittipong D, Tepparak N. Characteristics of behavioral and psychological symptoms of dementia, severity and levels of distress on caregivers. J Med Assoc Thai. 2014 Apr;97(4):423-30

8 Feast A, Moniz-Cook E, Stoner C, Charlesworth G, Orrell M. A systematic review of the relationship between behavioral and psychological symptoms (BPSD) and caregiver well-being. Int Psychogeriatr. 2016 Nov;28(11):1761-74.

9 Carvalho-Bos SS, Riemersma-van der Lek RF, Waterhouse J, Reilly T, Van Someren EJ. Strong association of the rest-activity rhythm with well-being in demented elderly women. Am J Geriatr Psychiatry. 2007 Feb;15(2):92-100.

10 Ancoli-Israel S, Cooke JR. Prevalence and comorbidity of insomnia and effect on functioning in elderly populations. J Am Geriatr Soc. 2005 Jul;53(7 Suppl):S264-71.

11 Giron MS, Forsell Y, Bernsten C, Thorslund M, Winblad B, Fastbom J. Sleep problems in a very old population: drug use and clinical correlates. J Gerontol A Biol Sci Med Sci. 2002 Apr;57(4):M236-40.

12 Potter GG, Steffens DC. Contribution of depression to cognitive impairment and dementia in older adults. Neurologist. 2007 May; 13(3):105-17.

13 Logsdon RG, McCurry SM, Teri L. Evidencebased interventions to improve quality of life for individuals with dementia. Alzheimers care today. 2007;8(4):309-18.

14 Wirz-Justice A, Werth E, Savaskan E, Knoblauch V, Gasio PF, Müller-Spahn F. Haloperidol disrupts, clozapine reinstates the circadian rest-activity cycle in a patient with earlyonset Alzheimer disease. Alzheimer Dis Assoc Disord. 2000 Oct-Dec;14(4):212-5.

15 van Straten A, van der Zweerde T, Kleiboer A, Cuijpers P, Morin CM, Lancee J. Cognitive and behavioral therapies in the treatment of insomnia: A meta-analysis. Sleep Med Rev. 2018 Apr;38:3-16.

16 Alessi CA, Martin JL, Webber AP, Cynthia Kim E, Harker JO, Josephson KR. Randomized, controlled trial of a nonpharmacological intervention to improve abnormal sleep/wake patterns in nursing home residents. J Am Geriatr Soc. 2005 May;53(5):803-10.

17 Martin JL, Webber AP, Alam T, Harker JO, Josephson KR, Alessi CA. Daytime sleeping, sleep disturbance, and circadian rhythms in the nursing home. Am J Geriatr Psychiatry. 2006 Feb;14(2):121-9.

18 Volkers KM, Scherder EJ. Impoverished environment, cognition, aging and dementia. Rev Neurosci. 2011;22(3):259-66.
19 Sánchez A, Millán-Calenti JC, Lorenzo-López L, Maseda A. Multisensory stimulation for people with dementia: a review of the literature. Am J Alzheimers Dis Other Demen. $2013 \mathrm{Feb} ; 28(1): 7-14$.

20 Maseda A, Cibeira N, Lorenzo-López L, González-Abraldes I, Buján A, de Labra C, et al. Multisensory Stimulation and Individualized Music Sessions on Older Adults with Severe Dementia: Effects on Mood, Behavior, and Biomedical Parameters. J Alzheimers Dis. 2018;63(4):1415-25.

21 Lorusso LN, Bosch SJ. Impact of multisensory environments on behavior for people with dementia: A systematic literature review. Gerontologist. 2018 May;58(3):e168-79.

22 Burns I, Cox H, Plant H. Leisure or therapeutics? Snoezelen and the care of older persons with dementia. Int J Nurs Pract. 2000 Jun;6(3):118-26.

23 Scherder E, Eggermont L, Visscher C, Scheltens $\mathrm{P}$, Swaab D. Understanding higher level gait disturbances in mild dementia in order to improve rehabilitation: 'last in-first out'. Neurosci Biobehav Rev. 2011 Jan;35(3):699-714.

24 Brun A, Gustafson L. Distribution of cerebral degeneration in Alzheimer's disease. A clinico-pathological study. Arch Psychiatr Nervenkr (1970). 1976 Dec;223(1):15-33.

25 van Hoof J, Kort HS, van Waarde H, Blom MM. Environmental interventions and the design of homes for older adults with dementia: an overview. Am J Alzheimers Dis Other Demen. 2010 May;25(3):202-32.

26 Shang B, Yin H, Jia Y, Zhao J, Meng X, Chen $L$, et al. Nonpharmacological interventions to improve sleep in nursing home residents: A systematic review. Geriatr Nurs. 2019 Jul Aug;40(4):405-16.

27 Strøm BS, Ytrehus S, Grov EK. Sensory stimulation for persons with dementia: a review of the literature. J Clin Nurs. 2016 Jul;25(13-14): 1805-34.

28 Smith BC, D'Amico M. Sensory-Based Interventions for Adults with Dementia and $\mathrm{Alz}$ heimer's Disease: A Scoping Review. Occup Ther Health Care. 2019 May:1-31.

29 Higgins JP, Green S, editors. Cochrane Handbook for Systematic Reviews of Interventions. London: Cochrane Collaboration; 2008.

30 Sterne JA, Hernán MA, Reeves BC, Savović J, Berkman ND, Viswanathan M, et al. ROBINS-I: a tool for assessing risk of bias in nonrandomised studies of interventions. BMJ. 2016 Oct;355:i4919.

31 Buettner LL, Fitzsimmons S. AD-venture program: therapeutic biking for the treatment of depression in long-term care residents with dementia. Am J Alzheimers Dis Other Demen. 2002 Mar-Apr;17(2):121-7.

32 Burns A, Allen H, Tomenson B, Duignan D, Byrne J. Bright light therapy for agitation in dementia: a randomized controlled trial. Int Psychogeriatr. 2009 Aug;21(4):711-21.

33 Edwards NE, Beck AM, Lim E. Influence of aquariums on resident behavior and staff satisfaction in dementia units. West J Nurs Res. 2014 Nov;36(10):1309-22.
34 Figueiro MG, Plitnick BA, Lok A, Jones GE, Higgins $P$, Hornick TR, et al. Tailored lighting intervention improves measures of sleep, depression, and agitation in persons with $\mathrm{Alz}$ heimer's disease and related dementia living in long-term care facilities. Clin Interv Aging. 2014 Sep;9:1527-37.

35 Fontana Gasio P, Kräuchi K, Cajochen C, Someren E, Amrhein I, Pache M, et al. Dawndusk simulation light therapy of disturbed circadian rest-activity cycles in demented elderly. Exp Gerontol. 2003 Jan-Feb;38(1-2):207-16.

36 Harris M, Richards KC, Grando VT. The effects of slow-stroke back massage on minutes of nighttime sleep in persons with dementia and sleep disturbances in the nursing home: a pilot study. J Holist Nurs. 2012 Dec;30(4):255-63.

37 Ito T, Yamadera H, Ito R, Suzuki H, Asayama K, Endo S. Effects of vitamin B12 on bright light on cognitive and sleep-wake rhythm in Alzheimer-type dementia. Psychiatry Clin Neurosci. 2001 Jun;55(3):281-2.

38 Kwok T, Leung PC, Wing YK, Ip I, Wong B, Ho DW, et al. The effectiveness of acupuncture on the sleep quality of elderly with dementia: a within-subjects trial. Clin Interv Aging. 2013;8:923-9.

39 van Lieshout-van Dal E, Snaphaan LJAE, Arkink N, Bongers IMB. Exposing people with dementia to biodynamic light. J Psychiatry Cogn Behav. 2018;3.

40 Lyketsos CG, Lindell Veiel L, Baker A, Steele C. A randomized, controlled trial of bright light therapy for agitated behaviors in dementia patients residing in long-term care. Int $\mathrm{J}$ Geriatr Psychiatry. 1999 Jul;14(7):520-5.

41 Mishima K, Okawa M, Hishikawa Y, Hozumi S, Hori H, Takahashi K. Morning bright light therapy for sleep and behavior disorders in elderly patients with dementia. Acta Psychiatr Scand. 1994 Jan;89(1):1-7.

42 Nowak L. The effect of timed blue-green light on sleep-wake patterns in women with Alzheimer's disease. Dissert Abstr Int B Sci Engin 2008;69:1-154

43 Onega LL, Pierce TW, Epperly L. Bright Light Therapy to Treat Depression in Individuals with Mild/Moderate or Severe Dementia. Issues Ment Health Nurs. 2018 May;39(5):370-3.

44 Riemersma-van der Lek RF, Swaab DF, Twisk J, Hol EM, Hoogendijk WJ, Van Someren EJ. Effect of bright light and melatonin on cognitive and noncognitive function in elderly residents of group care facilities: a randomized controlled trial. JAMA. 2008 Jun;299(22): 2642-55.

45 Rodríguez-Mansilla J, González-López-Arza MV, Varela-Donoso E, Montanero-Fernández J, Jiménez-Palomares M, Garrido-Ardila EM. Ear therapy and massage therapy in the elderly with dementia: a pilot study. J Tradit Chin Med. 2013 Aug;33(4):461-7.

46 Satlin A, Volicer L, Ross V, Herz L, Campbell S. Bright light treatment of behavioral and sleep disturbances in patients with Alzheimer's disease. Am J Psychiatry. 1992 Aug; 149(8):1028-32. 
47 Suzuki M, Tatsumi A, Otsuka T, Kikuchi K, Mizuta A, Makino K, et al. Physical and psychological effects of 6-week tactile massage on elderly patients with severe dementia. Am J Alzheimers Dis Other Demen. 2010 Dec; 25(8):680-6.

48 Wahnschaffe A, Nowozin C, Rath A, Floessner T, Appelhoff S, Münch M, et al. Nighttime activity forecast by season and weather in a longitudinal design - natural light effects on three years' rest-activity cycles in nursing home residents with dementia. Int Psychogeriatr. 2017 Dec;29(12):2071-80.

49 Witting W, Kwa IH, Eikelenboom P, Mirmiran $\mathrm{M}$, Swaab DF. Alterations in the circadian restactivity rhythm in aging and Alzheimer's disease. Biol Psychiatry. 1990 Mar;27(6):563-72.

50 Cummings JL, Mega M, Gray K, RosenbergThompson S, Carusi DA, Gornbein J. The Neuropsychiatric Inventory: comprehensive assessment of psychopathology in dementia. Neurology. 1994 Dec;44(12):2308-14.

51 Ray WA, Taylor JA, Lichtenstein MJ, Meador KG. The nursing home behavior problem scale. J Gerontol. 1992 Jan;47(1):M9-16.

52 Reisberg B, Borenstein J, Franssen E, Salob S, Steinberg G, Shulman E, et al. BEHAVE-AD: A clinical rating scale for the assessment of pharmacologically remediable behavioral symptomatology in Alzheimer's disease; Alzheimer's Disease. Springer; 1987. pp. 1-16.

53 Buysse DJ, Reynolds CF 3rd, Monk TH, Berman SR, Kupfer DJ. The Pittsburgh Sleep Quality Index: a new instrument for psychiatric practice and research. Psychiatry Res. 1989 May;28(2):193-213.

54 Onega LL, Pixley JL. Psychometric properties of the Depressive Symptom Assessment for Older Adults in individuals with moderate or severe dementia. Issues Ment Health Nurs. 2008 Sep;29(9):942-58.

55 Borenstein M, Rothstein $\mathrm{H}$. Comprehensive meta-analysis. Biostat; 1999.

56 Dowling GA, Hubbard EM, Mastick J, Luxenberg JS, Burr RL, Van Someren EJ. Effect of morning bright light treatment for rest-activity disruption in institutionalized patients with severe Alzheimer's disease. Int Psychogeriatr. 2005 Jun;17(2):221-36.

57 Dowling GA, Mastick J, Hubbard EM, Luxenberg JS, Burr RL. Effect of timed bright light treatment for rest-activity disruption in institutionalized patients with Alzheimer's disease. Int J Geriatr Psychiatry. 2005 Aug;20(8):738-43.

58 Dowling GA, Burr RL, Van Someren EJ, Hubbard EM, Luxenberg JS, Mastick J, et al. Melatonin and bright-light treatment for rest-activity disruption in institutionalized patients with Alzheimer's disease. J Am Geriatr Soc. 2008 Feb;56(2):239-46.

59 Sloane PD, Williams CS, Mitchell CM, Preisser JS, Wood W, Barrick AL, et al. Highintensity environmental light in dementia: effect on sleep and activity. J Am Geriatr Soc. 2007 Oct;55(10):1524-33.

60 Rücker G, Cates CJ, Schwarzer G. Methods for including information from multi-arm trials in pairwise meta-analysis. Res Synth Methods. 2017 Dec;8(4):392-403.

61 Morris SB, DeShon RP. Combining effect size estimates in meta-analysis with repeated measures and independent-groups designs. Psychol Methods. 2002 Mar;7(1):105-25.

62 Egger M, Davey Smith G, Schneider M, Minder C. Bias in meta-analysis detected by a simple, graphical test. BMJ. 1997 Sep;315(7109):629-34.

63 Duval S, Tweedie R. Trim and fill: a simple funnel-plot-based method of testing and adjusting for publication bias in meta-analysis. Biometrics. 2000 Jun;56(2):455-63.

64 Moher D, Liberati A, Tetzlaff J, Altman DG; PRISMA Group. Preferred reporting items for systematic reviews and meta-analyses: the PRISMA statement. PLoS Med. 2009 Jul; 6(7):e1000097.

65 Folstein MF, Folstein SE, McHugh PR. "Minimental state". A practical method for grading the cognitive state of patients for the clinician. J Psychiatr Res. 1975 Nov;12(3):189-98.

66 Moyle W, Jones C, Murfield J, Thalib L, Beattie E, Shum D, et al. Effect of a robotic seal on the motor activity and sleep patterns of older people with dementia, as measured by wearable technology: A cluster-randomised controlled trial. Maturitas. 2018 Apr;110:10-7.

67 Svansdottir HB, Snaedal J. Music therapy in moderate and severe dementia of Alzheimer's type: a case-control study. Int Psychogeriatr. 2006 Dec;18(4):613-21.

68 Alparslan GB, Özkaraman A, Özbabalık D, Çolak E. The Effect of Light on Daily Life Activities and Sleep in Patients with Alzheimer's Disease. J Turkish Sleep Med. 2019;6(3):59-64.

69 Mishima K, Hishikawa Y, Okawa M. Randomized, dim light controlled, crossover test of morning bright light therapy for rest-activity rhythm disorders in patients with vascular dementia and dementia of Alzheimer's type. Chronobiol Int. 1998 Nov;15(6):647-54.

70 Van Someren EJ, Kessler A, Mirmiran M, Swaab DF. Indirect bright light improves circadian restactivity rhythm disturbances in demented patients. Biol Psychiatry. 1997 May;41(9):955-63.

71 Van Someren EJ. Improving actigraphic sleep estimates in insomnia and dementia: how many nights? J Sleep Res. 2007 Sep;16(3):269-75.

72 Salami O, Lyketsos C, Rao V. Treatment of sleep disturbance in Alzheimer's dementia. Int J Geriatr Psychiatry. 2011 Aug;26(8):771-82.

73 Hadi K, Du Bose JR, Choi Y-S. The Effect of Light on Sleep and Sleep-Related Physiological Factors Among Patients in Healthcare Facilities: A Systematic Review. HERD. 2019 Oct;12(4):116-41.

74 van Maanen A, Meijer AM, van der Heijden $\mathrm{KB}$, Oort FJ. The effects of light therapy on sleep problems: A systematic review and metaanalysis. Sleep Med Rev. 2016 Oct;29:52-62.

75 Faulkner SM, Bee PE, Meyer N, Dijk DJ, Drake RJ. Light therapies to improve sleep in intrinsic circadian rhythm sleep disorders and neuro-psychiatric illness: A systematic review and meta-analysis. Sleep Med Rev. 2019 Aug;46:108-23.
76 Forbes D, Blake CM, Thiessen EJ, Peacock S, Hawranik P. Light therapy for improving cognition, activities of daily living, sleep, challenging behaviour, and psychiatric disturbances in dementia. Cochrane Database Syst Rev. 2014 Feb 26;(2):CD003946

77 Dimitriou TD, Tsolaki M. Evaluation of the efficacy of randomized controlled trials of sensory stimulation interventions for sleeping disturbances in patients with dementia: a systematic review. Clin Interv Aging. 2017 Mar;12:543-8.

78 McCurry SM, Logsdon RG, Teri L, Vitiello MV. Sleep disturbances in caregivers of persons with dementia: contributing factors and treatment implications. Sleep Med Rev. 2007 Apr;11(2):143-53.

79 Aalten P, de Vugt ME, Jaspers N, Jolles J, Verhey FR. The course of neuropsychiatric symptoms in dementia. Part I: findings from the two-year longitudinal Maasbed study. Int J Geriatr Psychiatry. 2005 Jun;20(6):523-30.

80 Safi A, Hodgson N. Timing of activities and their effects on circadian rhythm in the elderly with dementia: A literature review. J Sleep Disord Ther. 2014;3(5):1-5.

81 Van Someren EJ, Swaab DF, Colenda CC, Cohen W, McCall WV, Rosenquist PB. Bright light therapy: improved sensitivity to its effects on rest-activity rhythms in Alzheimer patients by application of nonparametric methods. Chronobiol Int. 1999 Jul;16(4):505-18.

82 Hoekert M, der Lek RF, Swaab DF, Kaufer D, Van Someren EJ. Comparison between informant-observed and actigraphic assessments of sleep-wake rhythm disturbances in demented residents of homes for the elderly. Am J Geriatr Psychiatry. 2006 Feb;14(2):104-11.

83 Ancoli-Israel S, Martin JL, Kripke DF, Marler M, Klauber MR. Effect of light treatment on sleep and circadian rhythms in demented nursing home patients. J Am Geriatr Soc. $2002 \mathrm{Feb} ; 50(2): 282-9$.

84 Ancoli-Israel S, Martin JL, Gehrman P, Shochat T, Corey-Bloom J, Marler M, et al. Effect of light on agitation in institutionalized patients with severe Alzheimer disease. Am J Geriatr Psychiatry. 2003 Mar-Apr;11(2):194-203.

85 Ancoli-Israel S, Gehrman P, Martin JL, Shochat T, Marler M, Corey-Bloom J, et al. Increased light exposure consolidates sleep and strengthens circadian rhythms in severe Alzheimer's disease patients. Behav Sleep Med. 2003;1(1):22-36.

86 Fetveit A, Skjerve A, Bjorvatn B. Bright light treatment improves sleep in institutionalised elderly-an open trial. Int J Geriatr Psychiatry. 2003 Jun;18(6):520-6.

87 Fetveit A, Bjorvatn B. The effects of brightlight therapy on actigraphical measured sleep last for several weeks post-treatment. A study in a nursing home population. J Sleep Res. 2004 Jun;13(2):153-8.

88 Takeda A, Watanuki E, Koyama S. Effects of Inhalation Aromatherapy on Symptoms of Sleep Disturbance in the Elderly with Dementia. Evid Based Complement Alternat Med. 2017;2017:1902807. 